\title{
A review on application of biomarkers in the field of bioinformatics \& nanotechnology for individualized
} cancer treatment

\begin{abstract}
In the current era of post genomics, research in discovery of biomarker with respect to the application of principles in nanotechnology and bioinformatics raised opportunities in the field of personalized medicine in which diseases are detected, diagnosed and regular follow up of therapeutic regimen were tailored to each and every individual on the basis of their molecular profile. In Case of predictive medicine the usage of genetic/molecular information play a vital role in predicting the development of disease progression and clinical outcome. In this review, we discuss the recent advancement of tools developed on the principles of bioinformtics to accelerate the biomarker discovery of cancer and the usage of multiplexed nanoparticle probes for profiling the biomarker of cancer. Finally, with respect to future prospectives and further challenges in biomarker discover of Cancer, we correlate the signatures of biomolecules with their clinical outcome. So, the term Bio-Nano-Info is a promise for individualized therapy with respect to molecular diagnosis of cancer and the same principle can also be applied to other human diseases.
\end{abstract}

Volume 5 Issue $6-2017$

\author{
Harishchander Anandaram \\ Department of Bioinformatics, Sathyabama University, India
}

Correspondence: Department of Bioinformatics, Sathyabama University, Chennai, India, Email harishchander.a@gmail.com

Received: April 04, 2017| Published: July 13, 2017

\section{Introduction}

In the Current era of post genomics, there is an exponential growth in the area of genomics and proteomics data and hence there was a major advancement in the field of understanding the molecular mechanisms of complex diseases in humans. There raised an increase in pace to develop new technologies to diagnose the molecular signatures of complex diseases to initiate the therapy with respect to the principle of personalized medicine and hence the new era of molecular medicine laid the path for detection of disease, diagnosis of complex disorders and performing treatment with respect to the molecular profile of each and every individual. ${ }^{1-4}$ This revolution had laid the foundation for the availability and application of new and novel biomarkers to predict the behavior of disease using advanced technologies to perform rapid diagnosis and detection. The evolutions of modern therapies to target cells were based on the principles of biocomputing. In order to address the molecular profiling and diagnostics, a major challenge was raised in characterization by histologic lesions in complex disorders because those lesions are heterogeneous at the cellular and molecular levels. In cancerous tumors, malignant cells are intermixed with blood vessels, stroma, and inflammatory cells. ${ }^{5-8}$ Current technologies like Gene microarrays and Real-Time Polymerase Chain Reactions (RTPCR) were not designed to handle the heterogeneic nature of cancer lesions and hence the development of nanotechnology provided a new opportunity to integrate morphological profile of diseased contion and molecular signatures and it is also used for correlating the observed cellular and molecular changes with respect to the behavior of pathological condition in complex disorders..$^{9-11}$ In particular, the application of bio conjugated quantum dots (QDs) $)^{12-15}$ quantifies the presence of multiple biomarkers in intact tissue specimens and cancer cells and it allows us to conduct a comparative test between traditional histopathology V.S. molecular signatures of the same tissue. ${ }^{16-20}$ Nanotechnology is being used in the fields of molecular imaging and therapy and it can be also be used to improve the toxicity and efficacy profiles of chemotherapeutic agents because these agents can be covalently attached or encapsulated. ${ }^{21-23}$
The present major task in biomedical nanotechnology is to understand the interaction of nanoparticles with cells, organs and blood under the physiological conditions in vivo and the need to overcome their limitations with respect to their delivery to organs or diseased target sites. ${ }^{24-26}$ The next major challenge is to generate a series of critical studies to identify the clear link between biomarkers VS disease behaviors like the rate of progression in tumor and their different responses with respect to radiation, drug therapy or surgery. ${ }^{27}$ In this context, we discuss the ways and level of integration of biomarkers and bio computing with nanotechnology to perform high-throughput analysis of gene expression data. We also discuss the application of web-based bioinformatics tools for the discovery, optimization and clinical validation of biomarker.

\section{Biomarker}

Biomarker or Bio-molecular markers include mutant or genes, proteins, RNA, carbohydrates, lipids, small metabolites and the altered expression states of such markers can be correlated with respect to a clinical outcome or biological behavior. ${ }^{28-31}$ Most of the biomarkers were discovered by the molecular profiling studies based on correlation or association between a disease behavior and molecular signature. The first study on molecular profiling of complex diseases was reported by Golub et al. ${ }^{32}$ and the outcome of this study helped in identifying the gene expression patterns that could classify tumors and it served as a base for yielding a new and novel insights into tumor pathology such as grade, stage, response to treatment and further clinical course. Gene expression studies further revealed the fact that the molecular signatures of each and every tumor as an outcome of the combined stromal, tumoral and inflammatory factors of the original heterogeneous lesion..$^{33}$ The initial correlation of gene expression patterns with clinical outcome was first reported for the diffusion of large B-cell lymphoma, ${ }^{34}$ a heterogeneous disease in clinical conditions. Whereas, most patients responded well to therapy had a prolonged survival. This variability in progression of disease can also be correlated with a distinct pattern of gene expression. The 
concept of identifying a specific molecular portrait for a specific tumor in each patient was validated in later stage by Bittner, Perou and their coworkers by an array of clinical samples. ${ }^{35,36}$ Recent work in several groups identified a unique gene expression pattern to correlate with the clinical outcomes various tumors which includes lung, breast, prostate and liver cancers. ${ }^{37-41}$

Biomarkers are divided into 3 categories: Predictive, Prognostic and therapeutic response. Predictive biomarkers identify the probability of patients benefit with respect to a particular treatment. For example, breast cancer patients with HER2 (ERBB2) gene encodes tyrosine kinase receptor were expected to benefit from trastuzumab (Herceptin) treatment. ${ }^{42}$ Prognostic biomarkers allow the prediction of natural course needed for individual cancer and it can distinguish between aggressive tumors and indolent tumors. In case of gene encoding the estrogen receptor is over expressed by the tumor, the patients might be a better candidate to respond tamoxifen treatment. ${ }^{43}$ Pharmacodynamic biomarkers measure the short-term treatment effects of a drug has on tumor and these were used to guide the selection of dosage in the earlier stages of clinical development of new drugs from lead molecules.

In most cases, single biomarkers failed to provide the required sensitivity and specificity with respect to the substantial heterogeneity in various type of cancer. It is not realistic to expect a single biomarker to provide information about the tissue type and malignant transformation in the various stages of tumor progression and development. Hence, panels of biomarkers are required for diagnosis. The discovery and validation of biomarkers must be subjected to several key steps before their application in clinical practice. Here, the initial step involves the acquisition and experimental design of molecular data, i.e. large amount of proteomic or genomic expression data together with the case history of patient. The data need to be properly annotated and organized using the available web-based tools and databases. Further, the original data were improved and evaluated by combining multiple datasets to increase the statistical significance. In the second stage of data processing the concept of feature extraction and classification principles were used to identify the relevant biomarkers, which are differentially expressed. Prior to the clinical application of these biomarkers, their functional relevance is validated by determining their level of expression with the application of RTPCR (for nucleic acids) or multiplexed nanotechnology (for proteins). Hence, we elaborate on the web-based application of bioinformatics tools for the analysis of microarray data to initiate the discovery of biomarker and analyze their validation in clinical studies.

\section{Bioinformatics}

In the final stage of pre-genomic era, microarray data analysis using bioinformatics tools focused on the concept of unsupervised clustering technique (Eg. Kohonen Map and Self Propagation based learning) in "Machine Learning" and the initiative was to explore new technologies and discover new properties within the structure of expression data by neglecting the normalized dwelling factors for application in potential clinical studies. For example, Eisen et al., ${ }^{44}$ had developed a software application that combines several types of unsupervised clustering methods. A more recent development was the combination of clustering algorithms and visualization tools into a web-based application ${ }^{45}$ with a focus on unsupervised clustering method. Similar methods have been applied to analyze the high-throughput data of gene expression from different clinical scenarios and certain significant findings from these tools lead to the identification of cancer subtypes. ${ }^{46,47}$ As such, applications developed by the principle of unsupervised clustering are still used for visualization of expression data and biomarker discovery.

Recently, the major focus of microarray data analysis has shifted from unsupervised clustering to and supervised analysis (For Example Back Propagation Algorithm, Support Vector Machine and etc.). Consequently, a web-based application of bioinformatics shifted towards the development of new tools for the analysis of genes which are differentially expressed under the known conditions. Some of these tools are specific to the platforms of microarray (For example, ILOOP (Interwoven Loop) and MAGMA were web based applications designed for analyzing the two-channel microarrays). ${ }^{48,49}$ ILOOP is an interface to assist the experimental design of two-channel microarrays and MAGMA incorporates the standard normalization procedure for converting statistical methods into an application for usability and reproducibility. Most of these web-based applications were implemented functionally to incorporate several common steps in the pipeline of data analysis. GEPAS (Gene Expression Profile Analysis Suite) includes the principles of data normalization, feature selection, class prediction, and unsupervised/supervised clustering. ${ }^{50}$

CARMA web (comprehensive R and bioconductor-based web) is another tool which was recently developed to perform the microarray data analysis, ${ }^{51}$ it uses several modules from Bioconductor (An open source bioinformatics software package incorporated in the $\mathrm{R}$ programming language-https:// carmaweb.genome.tugraz.at). The functions of microarray data analysis were available in Bio conductor and it includes the concept of background correction, quality control, normalization, differential gene detection, clustering, dimensionality reduction, and visualization. ${ }^{53}$ In bioinformatics application, the main contribution of CARMAweb is to integrate the numerous tools into a user-friendly interface in web. Gene Pattern ${ }^{53}$ is another tool to compile the analysis of different gene expression tools to reproduce integration in the cancer Bioinformatics Grid (caBIG), an initiative taken by the National Cancer Institute (NCI), to create a standard for bioinformatics software. ${ }^{54}$

It is a well established fact that, the candidate biomarkers were obtained from the outcome of microarray data analysis and they depend on the available samples and the selection algorithm. ${ }^{55} \mathrm{In}$ fact, these biomarkers can also be highly unstable and often varies from sample to sample. Furthermore, platforms with highthroughput assay can handle ten thousand of genes and most of the assays were not completely understood. Hence, the task of interpreting their results needs improvisation in statistics. By associating each candidate gene with a biological function, one might be able to understand the underlying mechanisms of the associated disease and their biological relevance on the basis of the algorithm for feature selection. Databases such as the Gene Ontology (GO) were designed to facilitate the interpretation of gene functions in large scale. ${ }^{56} \mathrm{~A}$ diverse range of GO tools were also available to extract the statistically significant conclusions from the analysis of GO database. Modules for GO analysis are either web-based or downloadable packages which includes GoMiner, ${ }^{57,58}$ GOStat, ${ }^{59} \mathrm{AmiGO},{ }^{60} \mathrm{BiNGO}^{61}$ and GOEAST. ${ }^{62}$ There are similar applications that mine the literature. CoPub links the lists of candidate genes to keywords that are obtained by searching literature in Medline abstracts and visualizes the keywords that are overrepresented using a network structure and the web address is http://services.nbic.nl/cgi-bin/copub/CoPub.pl. ${ }^{63}$

Since there is an increase in the accumulation of gene expression data, certain applications have emerged with the objective to organize and integrate the heterogeneous datasets from various data sources in 
an effective manner. As it was mentioned previously, the exponential increase in the sample size of data can improve the process of reproducibility in predictive models. Thus, there raised a demand for solutions to allow data sharing. Gene Expression Omnibus $(\mathrm{GEO})^{64}$ and Array Express ${ }^{65}$ were the examples of large warehouses (repositories) to manage the the community of data standards such as MIAME (Minimum Information about a Microarray Experiment). ${ }^{66}$ An alternative solution, ArrayWiki can allow the community of users to annotate gene expression metadata. ${ }^{67}$ Another initiative, caArray is a part of the caBIG initiative to become an interoperable standard for the storage of microarray in caBIG applications. ${ }^{54}$ There is an overlap between the analytical methods used in gene expression analysis and gene interpretation software. These overlaps are deposited in the data repositories using high-throughput methods. Further, a web-based application called the 'Microarray Retriever' ${ }^{68}$ was developed to retrieve the gene expression data from GEO and Array Express repositories for maximizing the potential of large-sample in microarray studies. Similarly, GEO meta $\mathrm{db}$ is an improvement for increasing the querying capabilities in GEO repository. ${ }^{69}$ Although this application available only for GEO, it is anticipated that it can also be applied to meta-analysis applications to increase the usefulness of repositories.

In spite of the availability of software packages, it is still difficult to use the output of data after the process of normalization and quality without the application of a subsequent clustering feature for selection..$^{70}$ Further, there is a need to translate the gene symbols on the basis of feature selection, prior to the interpretation of a particular GO application. Workflow applications, such as Taverna and Gene Trail Express address the issue of feature selection in various ways. Gene Trail Express is a web-based portal that implements its own process of statistical analysis, normalization, interpretation and visualization modules based on common methods. ${ }^{71}$ Taverna is more general and builds workflows for certified web services certified by caBIG. ${ }^{72}$

In order to identify a biomarker using a Web-based bioinformatics resource, omniBioMarker was developed by Phan et al. ${ }^{73}$ In this software, biomarkers are identified through several steps which include normalization, quality control, feature selection, biological interpretation, GO validation, and clinical prediction. Since a single path is not enough for the identification of biomarker, a pipeline was developed to perform well for all possible datasets, ${ }^{74}$ unique analysis of these parameters must be applied specifically for each clinical problem. The computational layout of omniBiomarker addresses the concept of fine-tuning in each and every step in the pipeline with respect to a particular dataset or clinical problem on the basis of prior knowledge in biology. ${ }^{73}$ Biological knowledge overcomes the "increase of dimensionality" to stabilize the results by increasing the reproducibility of clinical prediction.

The initial step in the pipeline for biomarker identification is quality control. Due to the stochastic nature (randomness) of highthroughput data, it is important to analyze the quality of data, prior to further analysis. Moreover, the large quantity of high-throughput data requires the applications of specialized software. There are several existing applications to analyze the quality of data for microarrays within a population of samples. These applications may with respect to their complexity of model in usability and it ranging from web based downloadable software packages like RMA Express ${ }^{75}$ and $\mathrm{dChip}^{76}$ to web-based online portals such as caCorrect. ${ }^{77}$ Though the gene expression assays are generally reproducible ${ }^{78}$ their statistical artifacts were in smaller datasets and there rise a need for identifying the corrected data prior to further data analysis.

\section{Impact of Nanotechnology in clinical validation by multipled molecular analysis}

In case of large data sets (say $>100,000$ genes and proteins), computation tools should be used to select and optimize a small panel of biomarkers which predicts the patients outcome for therapeutic response. Conjugated Nanoparticles of antibodies can be designed with the purpose of targeted therapy and molecular diagnosis. In case of multiplexed QD probes, a selected biomarker panel of clinical specimens can be in needle biopsies and tissue microarrays. The use age of a minimum of five to a maximum of ten protein biomarkers have a significant impact in disease diagnosis and personalized treatment. In order to achieve these goals, Xing et al. ${ }^{18}$ have obtained a promising result for the molecular profiling of clinical paraffinembedded by fixed formalin (FFPE) specimens in prostate cancer. In this study, four conjugates of QD-antibodies were used to recognize and detect the four antigens which are responsible for causing tumor (the tumor-suppressor p53, the E3 ubiquitin ligase mdm-2, the zinc-finger transcription factor EGR-1 and the cyclin-dependent kinase inhibitor $\mathrm{p} 21 / \mathrm{CDN} 1 \mathrm{~A})$. These markers were known to vital in diagnosis of prostate cancer and they have also been correlated with the behavior of tumor. ${ }^{79,80}$ In case of molecular profiling, the results of QDs were consistent with results obtained by the fluorescence in situ hybridization (FISH) and traditional immunohistochemistry (IHC) and using human breast cancer cells. ${ }^{19}$ Finally, It is important to note that the classification of tumor with antigens which are expressed at low levels can also be subjective and therefore it requires an experienced observer to contribute a considerable amount of variations in clinical studies. In contrast, quantitative QD measurements allow the accurate and user-independent determination of tumor antigens for genes/ proteins that are expressed at low levels. Thus, the molecular profiling of quantitative QD can standardize the categorization of antigens on specimens. This factor is the key fundamental of management in breast cancer because the benefit of hormonal therapies and drug trastuzumab depends not only on the presence but also on the quantity of hormone or HER2 receptors. ${ }^{81-90}$

\section{Challenges}

In the mere the future, various directions are needed to carry out researches that are promising particularly in the application of biomedical science but still it requires additional effort with concentration to achieve success. The initial direction of research involves the design and development of nanoparticles by a single or multiple functionalities.

In case of applications in cancer and other conditions in medical field, the functions of nanoparticle includes the concept of imaging (exist either as single or dual-modality) and therapy via drug delivery or a combination of several drugs to target ligands.

By adding certain functions, nanoparticles can be designed to have novel properties for novel applications. For example, the binary nanoparticle with dual functions can be utilized for targeted therapy and molecular imaging.

Bioconjugated QDs with drug target and imaging functions can be used for the applications involved in molecular profiling.

In Contrast, the ternary nanoparticles that combine three functions can be designed for simultaneous imaging and targeted therapy.

The next stage of research must address the issues involved in optimizing the panels of biomarkers on the basis of quantitative 
molecular profiling in bioinformatics along with the help of nanotechnology. For example, probes in bioconjugated nanoparticle can predict the treatment response and clinical outcome of cancer behavior in personalized therapy.

The most important direction in future research of personalized medicine is to further investigate the process of distribution, excretion, metabolism, and pharmacodynamics of nanoparticle in the in-vivo studies of animal models. These studies will play a vital role in the development of nanoparticles for clinical applications in treating cancer.

\section{Acknowledgements}

None.

\section{Conflict of interest}

The author declares no conflict of interest.

\section{References}

1. Ginsburg GS, McCarthy JJ. Personalized medicine:revolutionizing drug discovery and patient care. Trends Biotechnol. 2001;19(12):491-496.

2. Little PFR, Williams RBH, Wilkins MR. Inter-individual variation in expression:a missing link in biomarker biology. Trends Biotechnol. 2008;27(1):5-10.

3. Jain KK. Personalized medicine. Curr Opinion Mol Ther. 2002;4(6):548558.

4. Allison M. Is personalized medicine finally arriving? Nat Biotechnol. 2008;26(5):509-517.

5. Hepper GH. Tumor heterogeneity. Cancer Res. 1984;44(6):2259-2265.

6. Liu AY, Roudier MP, True LD. Heterogeneity in primary and metastatic porstate cancer as defined by cell surface CD profile. Am J Path 2004;165(5):1543-1556.

7. Steeg PS. Heterogeneity of drug target expression among metastatic lesions: lessons from a breast cancer autopy program. Clin Cancer Res. 2008;14(12):3643-3645

8. Wu JM, Fackler MJ, Halushka MK, et al. Heterogeneity of breast cancer metastasis:comparison of therapeutic target expression and promoter methylation between primary tumors and their multifocal metastases Clin Cancer Res. 2008;14(7):1938-1946.

9. Ferrari M. Cancer nanotechnology: Opportunities and challenges. Nat Rev Cancer. 2005;5(3):161-171.

10. Wang X, Yang L, Zhuo GZ, et al. Application of nanotechnology in cancer therapy and imaging. CA Cancer J Clin. 2008;58(2):97-110.

11. Nie S, Xing Y, Kim GJ, et al. Nanotechnology applications in cancer Annu Rev Biomed Eng. 2007;9:257-288.

12. Chan WCW, Nie SM. Quantum dot bioconjugates for ultrasensitive nonisotopic detection. Science. 1998;281(5385):2016-2018.

13. Alivisatos AP. The use of nanocrystals in biological detection. Nat Biotechnol. 2001;22(1):47-52.

14. Michalet X, Pinaud FF, Bentolila LA, et al. Quantum dots for live cells, in vivo imaging, and diagnostics. Science. 2001;307(5709):538-544.

15. Gao XH, Yang L, Petros JA, et al. In-vivo molecular and cellular imaging with quantum dots. Curr Opin Biotechnol. 2001;16(1):63-72.

16. Gao XH, Nie SM. Molecular profiling of single cells and tissue specimens with quantum dots. Trends Biotechnol. 2001;21(9):371-373.
17. Xing Y, Smith AM, Agrawal A, et al. Molecular profiling of single cancer cells and clinical tissue specimens with semiconductor quantum dots. Int J Nanomedicine. 2006;1(4):473-4781.

18. Xing Y, Chaudry Q, Shen C, et al. Bioconjugated quantum dots for multiplexed and quantitative immunohistochemistry. Nat Protoc. 2007;2(5):1152-1165.

19. Yezhelyev MV, Al-Hajj A, Morris C, et al. In situ molecular profiling of breast cancer biomarkers with multicolor quantum dots. Adv Mat 2007;19(20):3146

20. Ghazani AA, Lee JA, Klostranec J, et al. High throughput quantification of protein expression of cancer antigens in tissue microarray using quantum dot nanocrystals. Nano Lett. 2006;6(12):2881-2886.

21. Yezhelyev M, Gao XH, Xing Y, et al. Emerging use of nanoparticles in diagnosis and treatment of breast cancer. Lancet Oncol. 2006;7(8):657767.

22. Sinha R, Kim GK, Nie SM, et al. Nanotechnology in cancer therapeutics: bioconjugated nanoparticles for drug delivery. Mol Cancer Ther. 2006;5(8):1909-1917.

23. Davis ME, Chen Z, Shin DM. Nanoparticle therapeutics: an emerging treatment modality for cancer. Nat Rev Drug Disc. 2008;7(9):771-782.

24. Jain RK. Transport of molecules, particles, and cells in solid tumors Annu Rev Biomed Eng. 1999;1:241-263.

25. Jain RK. Delivery of molecular and cellular Medicine to solid tumors Adv Drug Deliv Rev. 2001;46(1-3):149-168.

26. Jain RK. The next frontier of molecular medicine: Delivery of therapeutics. Nat Med. 1998;4(6):655-657.

27. Wang MD, Simons JW, Nie SM. Biomedical nanotechnology with bioinformatics - The promise and current progress. Proc IEEE. 2007;95(7):1386-1389.

28. Liotta L, Petricoin E. Molecular profiling of human cancer. Nature Reviews Genetics. 2000;1:48-56.

29. Petricoin EF, Zoon KC, Kohn EC, et al. Clinical proteomics: Translating benchside promise into bedside reality. Nat Rev Drug Discov. 2002;1(9):683-695.

30. Negm RS, Verma M, Srivastava S. The promise of biomarkers in cancer screening and detection. Trends Mol Med. 2002;8(6):288-293.

31. Ludwig JA, Weistein JN. Biomarkers in cancer staging, prognosis and treatment selection. Nat Rev Cancer. 2005;5(11):845-856.

32. Golub TR, Slonim DK, Tamayo P, et al. Molecular classification of cancer: Class discovery and class prediction by gene expression monitoring Science. 1999;286(5439):531-537.

33. Ross DT, Scherf U, Eisen MB, et al. Systematic variation in gene expression patterns in human cancer cell lines. Nat Genet. 2000;24(3):227-235.

34. Alizadeh AA, Eisen MB, Davis RE, et al. Distinct types of diffuse large B-cell lymphoma identified by gene expression profiling. Nature. 2000;403(6769):503-511.

35. Perou CM, Sorlie T, Eisen MB, et al. Molecular portraits of human breast tumours. Nature. 2000;406(6797):747-752

36. Bittner M, Meitzer P, Chen Y, et al. Molecular classification of cutaneous malignant melanoma by gene expression profiling. Nature. 2000;406(6795):536-540.

37. Dhanasekaran SM, Barrette TR, Ghosh D, et al. Delineation of prog nostic biomarkers in prostate cancer. Nature. 2001;412(6848):822-826.

38. Paik S, Shak S, Tang G, et al. A multigene assay to predict recurrence of tamoxifen-treated, nodenegative breast cancer. $N$ Engl J Med. 2004;351(27):2817-2826. 
39. Chen, Yu SL, Chen $\mathrm{CH}$, et al. A five-gene signature and clinical outcome in non-small-cell lung cancer. New Eng J Med. 2007;356(1):11-20.

40. Beer DG, Kardia SL, Huang CC, et al. Gene expression profiles predict survival of patients with lung adenocarcinoma. Nat Med. 2002;8(8):816824

41. Hoshida Y, Villanueva A, Kobayashi M, et al. Gene expression in fixed tissues and outcome in heptocellular carcinoma. New Eng J Med. 2008;359(19):1995-2004.

42. Hynes NE, Lane HA. ERBB receptors and cancer:The complexity of targeted inhibitors. Nat Rev Cancer. 2005;5(5):341-354.

43. Osborne CK. Tamoxifen in the treatment of breast cancer. New Eng $J$ Med. 1998;339(22):1609-1618.

44. Eisen M, Spellman PT, Brown PO, et al. Cluster analysis and display of genome-wide expression patterns. Proc Natl Acad Sci USA. 1998;95(25):14863-14868.

45. Geraci F, Pellegrini M, Renda M. AMIC@: All MIcroarray Clusterings @ once. Nucleic Acids Res. 1998;36:W315-W319.

46. de Souto M, Costa IG, de Araujo DS, et al. Cluster cancer gene expression data: a comparative study. BMC Bioinformatics. 2008;9:497.

47. de Revnies A, Assié G, Rickman DS, et al. Gene expression profiling reveals a new classification of adrenocortical tumors and identifies molecular predictors of malignancy and survival. J Clin Oncol. 2009;27(7):1108-1115.

48. Rehrauer H, Zoller S, Schlapbach R. MAGMA: analysis of two-channel microarrays made easy. Nuc Acids Res. 2007;35:W86-W90.

49. Pirooznia M, Gong P, Yang JY, et al. ILOOP-a web application for two-channel microarray interwoven loop design. BMC Genomics. 2008;9(Suppl 2):S11.

50. Tarraga J, Medina I, Carbonell J, et al. GEPAS, a web-based tool for microarray data analysis and interpretation. Nuc Acids Res. 2008;36:W308W314.

51. Rainer J, Sanchez-Cabo F, Stocker G, et al. CARMA web: comprehensive R- and bioconductor-based web service for microarray data analysis. Nuc Acids Res. 2006;34:W498-W503.

52. Gentleman R, Carey VJ, Bates DM, et al. Bioconductor: open software development for computational biology and bioinformatics. Genome Biol. 2004;5(10):R80.

53. Reich M, Liefeld T, Gould J, et al. Gene Pattern 2.0. Nature Genetics. 2006;38(5):500-501.

54. https://cabig.nci.nih.gov/

55. Michiels S, Koscielny S, Hill C. Prediction of cancer outcome with microarrays:a multiple random validation study. Lancet. 2005;365(9458):488-492.

56. Consortium TGO. Gene Ontology: tool for the unification of biology. Nature Genetics. 2000;25(1):25-29.

57. Zeeberg B, Feng W, Wang G, et al. GoMiner: a resource for biological interpretation of genomic and proteomic data. Genome Biol. 2003;4(4):R28.

58. Zeeberg B, Qin H, Narasimhan S, et al. High-Throughput GoMiner, an 'industrial-strength' integrative Gene Ontology tool for interpretation of multiple-microarray experiments, with application to studies of Common Variable Immune Deficiency (CVID). BMC Bioinformatics 6:168.

59. Beissbarth T, Speed T (2004) GOstat: Find statistically overrepresented Gene Ontologies within a group of genes. Bioinformatics. 2005;20(9):1464-1465.

60. Carbon S, Ireland A, Mungall CJ, et al. AmiGO: online access to ontology and annotation data. Bioinformatics. 2009;25(2):288-289.
61. Maere S, Heymans K, Kuiper M. BiNGO: a Cytoscape plugin to assess overrepresentation of gene ontology categories in Biological Networks. Bioinformatics. 2005;21(16):3448-3449.

62. Zheng Q, Wang X-J. GOEAST: a web-based software toolkit for Gene Ontology enrichment analysis. Nuc Acids Res. 2008;36:W358-W363.

63. Frijters R, Heupers B, van Beek P, et al. CoPub: a literature-based keyword enrichment tool for microarray data analysis. Nuc Acids Res. 2008;36:W406-W410.

64. Edgar R, Domrachev M, Lash AE. Gene Expression Omnibus: NCBI gene expression and hybridization array data repository. Nucleic Acids Res. 2002;30(1):207-210.

65. Parkinson H, Kapushesky M, Shojatalab M, et al. Array Express-a public database of microarray experiments and gene expression profiles. Nucleic Acids Res. 2006;35:D747-D750.

66. Brazma A, Hingamp P, Quackenbush J, et al. Minimum information about a microarray experiment (MIAME)-toward standards for microarray data. Nat Genet. 2001;29(4):365-371.

67. Todd H Stokes, JT Torrance, Henry Li, et al. Array Wiki: an enabling technology for sharing public microarray data repositories and metaanalysis. BMC Bioinformatics. 2008;9(Suppl 6):S18.

68. Ivliev A, t Hoen PA, Villerius MP, et al. Microarray retriever: a webbased tool for searching and large scale retrieval of public microarray data. Nuc Acids Res. 2008;36:W327-W331.

69. Zhu Y, Davis S, Stephens R, et al. GEO metadb: powerful alternative search engine for the gene expression omnibus. Bioinformatics. 2008;24(23):2798-2800.

70. Ochs M, Casagrande J. Information systems for cancer research. Cancer Invest. 2008;26(10):1060-1067.

71. Andreas Keller, Christina Backes, Maher Al-Awadhi, et al. Gene Trail Express: a web-based pipeline for the statistical evaluation of microarray experiments. BMC Bioinformatics. 2008;9:552.

72. Hull D, Katy Wolstencroft, Robert Stevens, et al. Taverna: a tool for building and running workflows of services. Nucl Acids Res. 2006;34:W729W732.

73. Phan J, Qiqin Yin-Goen, Andrew N Young, et al. Improving the efficiency of biomarker identification using biological knowledge. Pacific Symposium on Biocomputing. 2009;14:427-438.

74. Fogel G. Computational intelligence approaches for pattern discovery in biological systems. Brief Bioinform. 2008;9(4):307-316.

75. Irizarry R, Bolstad BM, Collin F, et al. Summaries of Affymetrix GeneChip probe level data. Nuc Acids Res. 2003;31(4):e15.

76. Li C. Automating dChip:toward reproducible sharing of microarray data analysis. BMC Bioinformatics. 2008;9:231

77. Stokes T, Moffitt RA, Phan JH, et al. chip artifact CORRECTion (caCORRECT): a bioinformatics system for quality assurance of genomics and proteomics array data. Ann Biomed Eng. 2007;35(6):1068-1080.

78. Shi L, Reid LH, Jones WD, et al. The MicroArray Quality Contro (MAQC) project shows inter- and intraplatform reproducibility of gene expression measurements. Nat Biotechnol. 2006;24(9):1151-1161.

79. Hernandez I, Maddison LA, Wei Y, et al. Prostate-specific expression of p53 (R172L) differentially regulates p21, Bax, and mdm2 to inhibit prostate cancer progression and prolong survival. Mol Cancer Res. 2003;1(14):1036-1047.

80. Mora GR, Olivier KR, Mitchell RF, et al. Regulation of expression of the early growth response gene-1 (EGR-1) in malignant and benign cells of the prostate. Prostate. 2005;63(2):198-207.

81. Marquez DC, Pietras RJ. Membrane-associated binding sites for estrogen contribute to growth regulation of human breast cancer cells. Oncogene. 2001;20(39):5420-5430. 
82. Hicks DG, Tubbs RR. Assessment of the HER2 status in breast cancer by fluorescence in situ hybridization:a technical review with interpretive guidelines. Hum Pathol. 2005;36(3):250-261.

83. Konecny G, Pauletti G, Pegram M, et al. Quantitative association be tween HER-2/neu and steroid hormone receptors in hormone receptorpositive primary breast cancer. J Natl Cancer Inst. 2003;95(2):142-153.

84. Bellman RE. Adaptive Control Processes. Princeton, USA: Princeton University Press; 1961.

85. Yuanhui Xiao, Robert Frisina, Alexander Gordon, et al. Multivariate search for differentially expressed gene combinations. BMC Bioinformatics. 2004;5:164.

86. Ding C, Peng H. Minumum redundancy feature selection from microarray gene expression data. J Bioin Comput Biol. 2005;3(2):185-205.
87. Hua J, Xiong Z, Lowey J, et al. Optimal number of features as a function of sample size for various classification rules. Bioinformatics. 2005;21(8):1509-1515.

88. Schuetz AN, Yin-Goen Q, Amin MB, et al. Molecular classification of renal tumors by gene expression profiling. J Mol Diagn. 2005;7(2):206218

89. Skubitz K, Zimmermann W, Kammerer R, et al. Differential gene expression identifies subgroups of renal cell carcinoma. J Lab Clin Med. 2006;147(5):250-267.

90. Rohan S, Tu JJ, Kao J, et al. Gene expression profiling separates chromophobe renal cell carcinoma from oncocytoma and identifies vesicular transport and cell junction proteins as differentially expressed genes. Clin Cancer Res. 2006;12(23):6937-6945. 